\title{
VARIATION OF SUPERPARAMAGNETIC PROPERTIES WITH IRON LOADING IN MAMMALIAN FERRITIN
}

\author{
Richard B. FRANKEL \\ Department of Physics, California Polvtechnic State University. San Luis Ohispo, CA 93407. U.S.A.
}

\author{
Georgia C. PAPAEFTHYMIOU
}

Francis Bitter National Magnet Laboratory, Massachusetts Institute of Technologi: Cambridge. MA 02139, U.S.A.

and Gerald D. WATT

Department of Chemistry, Brigham Young University. Provo, UT 84602, U.S.A.

The average blocking temperatures of ferritin molecules containing differing amounts of iron were determined by Mossbauer spectroscopy. The results imply that the magnetic anisotropy of the ferritin core particles is a function of particle volume. By addition of ${ }^{57} \mathrm{Fe}$ to ferritin core particles it was determined that, at a given temperature within the superparamagnetic temperature region, the "last-in" ferric ions have average relaxation times that are shorter than those of the bulk ferric ions.

\section{Introduction}

Mammalian ferritin is a roughly spherical, $130 \AA$ diameter protein composed of 24 subunits that form a shell enclosing a central cavity or core of $70 \AA$ diameter [1]. Up to 4500 iron atoms can be sequestered in the core in the form of a ferric-hydrous-oxide mineral particle associated with phosphate. The function of the protein is to provide cells with a readily mobilizable, biologically compatible form of iron necessary for various metabolic purposes while simultaneously binding iron strongly and thereby protecting cells from deleterious effects of free cellular iron. Similar proteins are found in a wide variety of plants and animals. and in bacteria [2-4].

Ferritin catalyzes the oxidation of ferrous iron by oxygen or other oxidants with deposition of the resulting ferric ions in the protein core [5]. A combination of statistical factors results in a distribution in the number of ferric ions per ferritin molecule. Mobilization of iron from the protein is accomplished by reduction of ferric ions in the core and chelation of the resulting ferrous ions by external chelators. The mechanisms and kinetics of these processes are currently under intense investigation. 
The structure of mammalian ferritin has been reported [1.5] at $2.8 \AA$ resolution. The subunits are arranged in pairs with 432 symmetry, and form channels leading from the exterior of the protein to the central core along 3-fold (8 channels) and 4-fold (6 channels) symmetry axes. The 4-fold channels are lined with leucine amino acid residues and are essentially hydrophobic. The 3 -fold channels are lined with carboxylate groups and are essentially hydrophyllic. These channels presumably play a significant role in the deposition and mobilization of iron from the ferritin core.

A variety of physical techniques including $X$-ray and electron diffraction [6,7], high resolution transmission electron microscopy [8,9]. EXAFS [10], EPR [11], NMR relaxometry [12,13], magnetic measurements [14-17], and Mössbauer spectroscopy $[10,14,18-26]$ have been used to study the properties of the ferric oxy-hydroxide core particles of ferritin. The particles consist of the quasicrystalline, ferric-hydrous-oxide mineral, ferrihydrite. The ferric ions in each particle are octahedrally coordinated by oxygen atoms and are in the high-spin $(S=5 / 2)$ state. Negative (antiferromagnetic) exchange interactions between the ferric ions in a particle result in a reduced effective paramagnetic moment per iron ion at room temperature and antiferromagnetic ordering of the ions in a particle at low temperature. The Néel temperature has been estimated to be $174 \mathrm{~K}$ [17]. Because the core particles are very small, the antiferromagnetically coupled sublattice magnetizations in a particle do not completely cancel and there is a small, permanent, magnetic dipole moment per particle below the Néel temperature. According to Néel [27], the number of uncompensated ions in a small, antiferromagnetically-ordered particle is $n^{1 / 2}$, where $n$ is the number of transition metal ions in the particle. The magnetic moment of a ferritin particle with 2000 ferric ions, each with magnetic dipole moment $5 \mu_{\mathrm{B}}$, would then be about $224 \mu_{\mathrm{B}}$. Because of the protein shells, the particles in adjacent molecules are at least $30 \AA$ apart and the interparticle interaction energy is low. Thus the ferritin core particles are superparamagnetic, or superantiferromagnetic. below the Néel temperature.

Superparamagnetism also accounts for the characteristic temperature dependence of the Mössbauer spectrum of ferritin in zero applied magnetic field [14]. Typically, the spectrum of ferritin at helium temperatures consists of a broadened magnetic sextet corresponding to a distribution of magnetic hyperfine fields with a mode of about $500 \mathrm{kOe}$ in the antiferromagnetically ordered particle. As the temperature is raised, a quadrupole doublet is superposed on the sextet, with the absorption intensity of the doublet and sextet increasing and decreasing, respectively, with increasing temperature. Above $80 \mathrm{~K}$, well below the Néel temperature of $174 \mathrm{~K}$, the spectrum consists of just the quadrupole doublet.

According to the theory of superparamagnetism applied to the Mössbauer spectra of small, magnetically ordered particles including ferritin [14,19,20,28,29], thermal energy can excite transitions of the sublattice magnetizations between energetically equivalent orientations (easy axes) in the particle with a frequency $\nu$ 
that is given by

$\nu=\nu_{0} \exp \left[-E_{\mathrm{A}} / k T\right]$

where $\nu_{0}$ is a constant characteristic of the material typically of the order of $10^{9}$ $\sec ^{-1}, E_{\mathrm{A}}$ is the magnetic anisotropy energy of the particle, and $k_{\mathrm{B}} T$ is the product of Boltzmann's constant and temperature. For a particle with uniaxial anisotropy, $E_{\mathrm{A}}$ is usually written

$E_{\mathrm{A}}=K V$,

where $K$ is the anisotropy energy per unit volume and $V$ is the core volume. For $\nu>\nu_{\mathrm{L}}$, the nuclear Larmor precession frequency of the ${ }^{57} \mathrm{Fe}$ nucleus in the local magnetic hyperfine field, the Mössbauer spectrum collapses to a quadrupole doublet. For $\nu<\nu_{L}$, the spectrum is the full magnetic hyperfine sextet. By definition, the blocking temperature $T_{\mathrm{B}}$ is the temperature at which $\nu=\nu_{\mathrm{L}}$. Because there is a distribution of particle volumes in each ferritin sample, there is a corresponding distribution of blocking temperatures; hence one usually refers to the average blocking temperature $\left\langle T_{\mathrm{B}}\right\rangle$.

In this paper, we report on the variation of the average blocking temperature in ferritin, determined by Mössbauer spectroscopy, due to variation in the average size of the ferritin core particle. This variation was accomplished by subjecting samples of ferritin to reduction and chelation to remove specified amounts of iron from the cores. We have found that the average blocking temperature, and hence the anisotropy energy, is not linear with particle volume, which implies that the anisotropy energy per unit volume $K$ is a function of particle volume. We have also studied the superparamagnetic properties of the "last-in" ferric ions, presumably on or near the surfaces of the ferritin core particles, by addition of

${ }^{57} \mathrm{Fe}$-enriched ferrous ions to ferritin, followed by oxidation. This experiment showed that the relaxation times that characterize these "last in" ferric ions are shorter than those reflected by the bulk average blocking temperature.

\section{Materials and methods}

Samples were prepared as previously described [30.31]. Horse spleen holoferritin was obtained from Sigma at a concentration of $100 \mathrm{mg} / \mathrm{ml}$ in $0.15 \mathrm{M} \mathrm{NaCl}$. The average iron content was determined to be 2100 iron atoms per ferritin molecule by the bathophenanthroline method. Ferritin samples with $20 \%$, 40\%, $60 \% 80 \%$ and $100 \%$ of the original $2100 \mathrm{Fe}^{3+} /$ molecule removed were prepared by: 1) reducing ferritin to the desired extent (i.e., $20 \%, 40 \%$, etc.) with $\mathrm{S}_{2} \mathrm{O}_{4}^{2-}$ containing $5 \times 10^{-5} \mathrm{M}$ methyl viologen (MV) for $30 \mathrm{~min}$; 2) adding excess bipyridyl (bipyd) and incubating an additional $30 \mathrm{~min}$; ; and 3) separating $\mathrm{Fe}$ (bipyd) ${ }_{3}^{2+}$ from ferritin on an anaerobic $1 \times 40 \mathrm{~cm}, \mathrm{G}-25$ Sephadex column. The emerging ferritin was free of $\mathrm{Fe}$ (bipyd) ${ }_{3}^{2+}$ and the ferritin bound iron was all in 
the $\mathrm{Fe}^{3+}$ state, as determined by coulometry and Mössbauer spectroscopy. These modified ferritin samples were concentrated, their protein concentrations made equal and their iron content determined. Sedimentation patterns for each protein sample $(10 \mathrm{mg} / \mathrm{ml}$ in $0.05 \mathrm{M}$ TES $0.1 \mathrm{M} \mathrm{NaCl} \mathrm{pH} \mathrm{7.5)} \mathrm{were} \mathrm{recorded} \mathrm{on} \mathrm{a}$ Beckman Model $\mathrm{E}$ ultracentrifuge under identical ultracentrifugation conditions of $50400 \mathrm{rpm}$ and $20^{\circ} \mathrm{C}$ [31].

${ }^{57} \mathrm{Fe}$-enriched ferritin samples were prepared by incubating holoferritin $(2100$ ferric ions per molecule) with ferrous ions prepared from $95 \%$ enriched ${ }^{57} \mathrm{Fe}$ iron metal under anaerobic conditions. The sample was oxidized by exposure to air following anaerobic Sephadex G-25 column chromatography to remove unbound ferrous ions [30]. Approximately $100{ }^{57} \mathrm{Fe}$ ferric ions were added to ferritin core particles each originally containing an average of $46{ }^{57} \mathrm{Fe}$ ferric ions (based on the $2.2 \%$ natural abundance of ${ }^{57} \mathrm{Fe}$ ). Thus Mössbauer spectroscopy of this sample was weighted toward the "last-in" ferric ions.

Mössbauer spectra were recorded with frozen ferritin solution samples in sealed plastic containers, using a constant acceleration spectrometer with a variable temperature dewar. Samples I-IV corresponded to 0\% (holoferritin). $20 \%, 40 \%$ and $60 \%$ iron removed from the protein, respectively. Sample V had $40 \%$ iron reduced but not removed from the protein. Sample VI had ${ }^{57} \mathrm{Fe}$-enriched ferric ions added to holoferritin.

\section{Results}

Mössbauer spectra of samples I to IV were similar to those reported previously for oxidized ferritin $[10,14,18-26]$. In each case the spectrum consisted of a broadened quadrupole doublet for $T \geqslant 80 \mathrm{~K}$, and a broadened magnetic hyperfine sextet for $T \leqslant 10 \mathrm{~K}$. Between 10 and $80 \mathrm{~K}$ the magnetic hyperfine sextet and quadrupole doublet were superposed, with intensities that decreased and increased, respectively, with increasing temperature. The magnetic hyperfine field distributions in the sextet spectra were similar for all four samples.

The temperature dependence of the spectra was associated with superparamagnetism of the ferritin iron core particles. We defined the magnetic fraction of the spectrum as the ratio of the intensity of the sextet spectrum to the total (sextet + quadrupole) spectral intensity. This quantity is plotted as a function of temperature for I. IV, and V in fig. 1. Data for II and III were similar but omitted for clarity. For samples I to IV, there was a monotonic decrease in the average blocking temperature $\left\langle T_{\mathrm{B}}\right\rangle$. defined as the temperature for which the magnetic fraction is 0.5 . as the average number of ferric ions per ferritin core decreased. $\left\langle T_{\mathrm{B}}\right\rangle$ is plotted as a function of the average number of iron atoms in the core of samples I to IV in fig. 2.

The spectrum of $\mathrm{V}$ was similar to those previously reported for partially reduced ferritin $[22,30]$. In addition to the ferric spectrum, consisting of a 


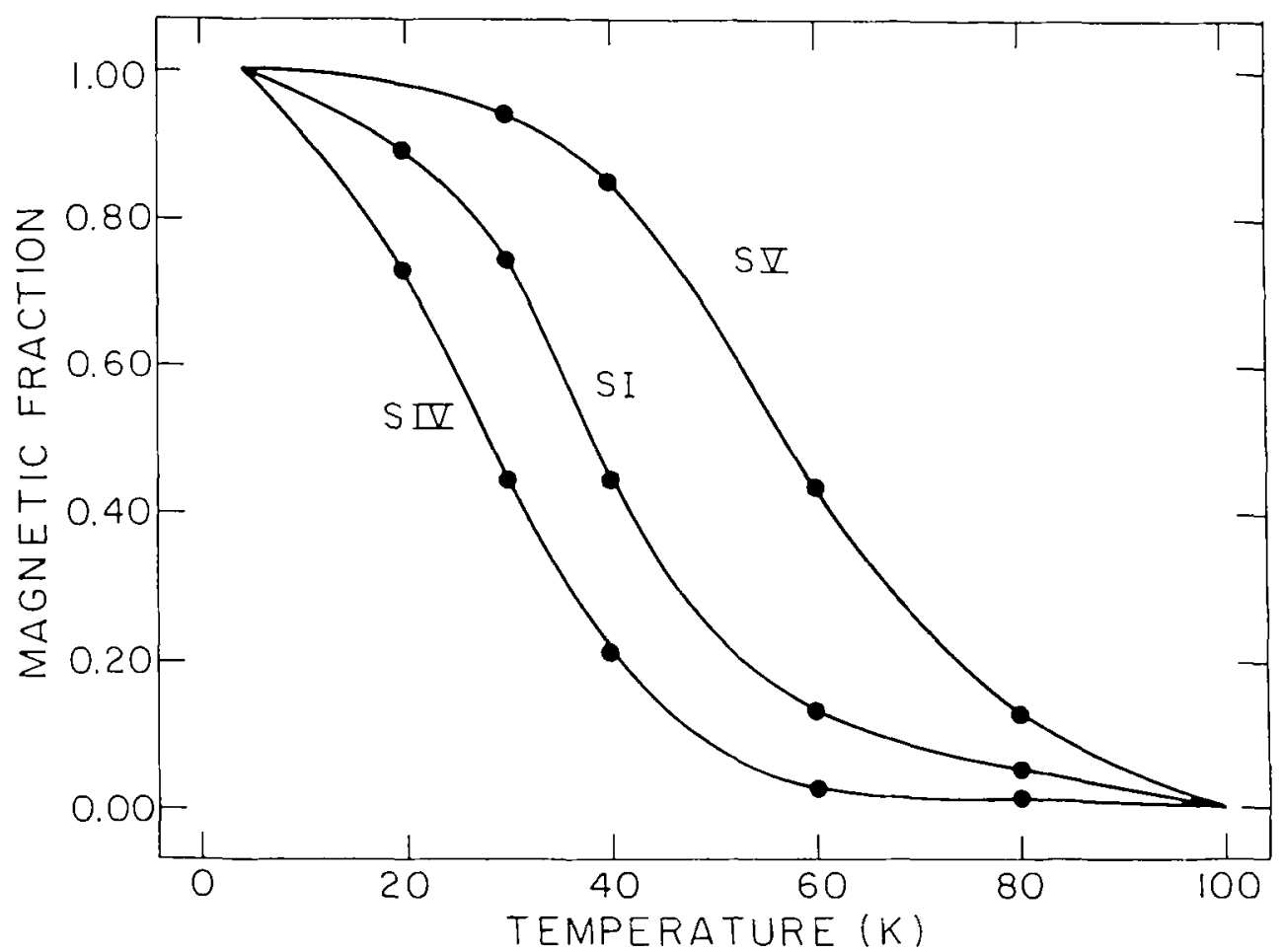

Fig. 1. Magnetic fraction, defined as the ratio of the intensity of the magnetic hyperfine sextet to the total intensity of the ferric spectrum, plotted as a function of temperature for samples I. IV and

$\mathrm{V}$. The blocking temperature is the temperature for which the magnetic fraction $=0.5$.

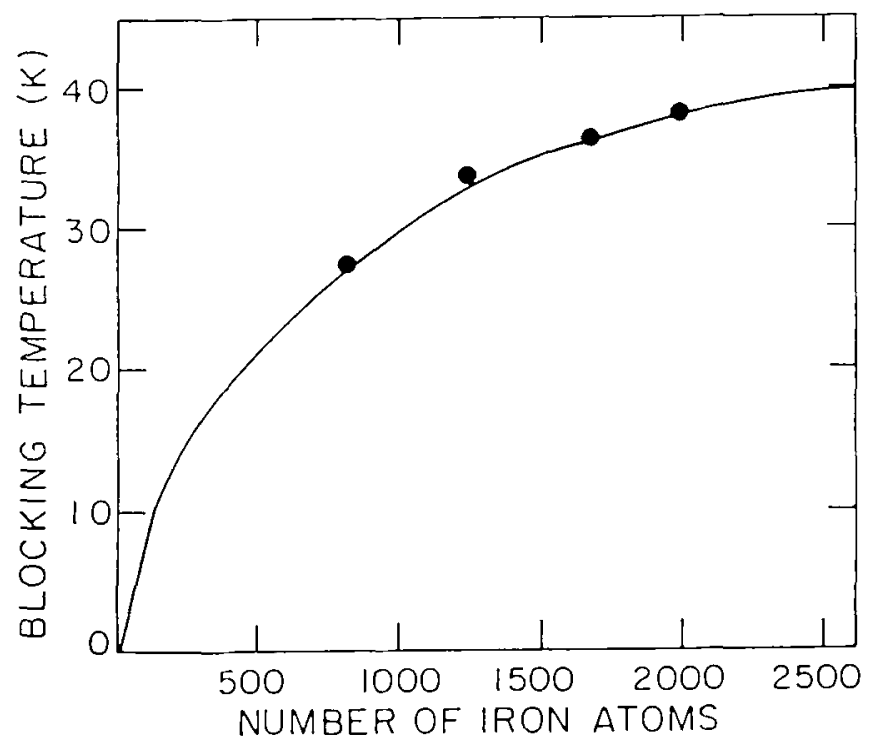

Fig. 2. Blocking temperatures plotted as a function of the number of iron atoms in the ferritin core particles for samples I to IV. The solid line is theoretical assuming different contributions of bulk and surface ferric ions to the magnetic anisotropy energy (eqs. (4) and (5)). 


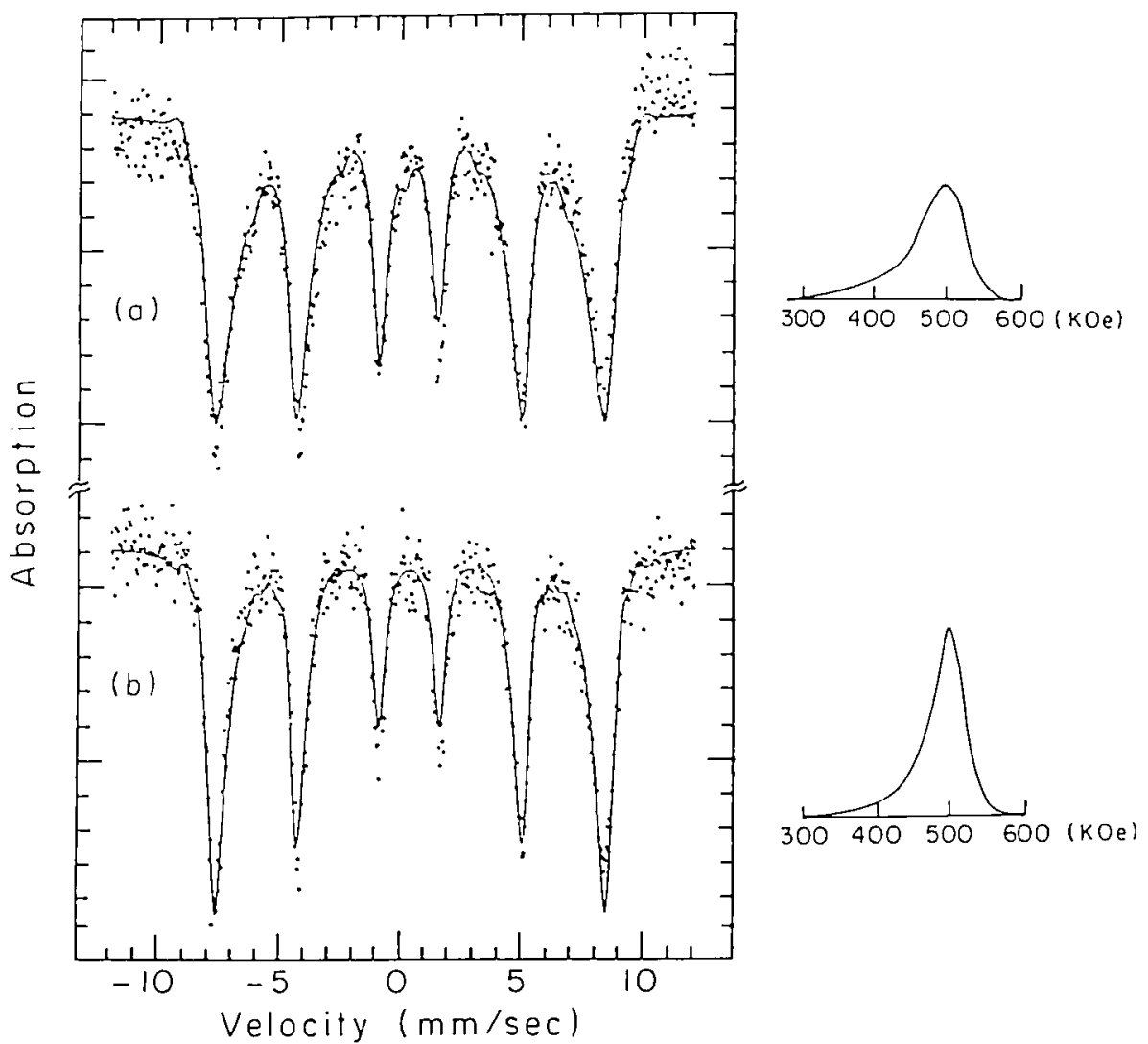

Fig. 3. Mössbauer spectra at $2 \mathrm{~K}$ for: (a) holoferritin following incubation with ${ }^{57} \mathrm{Fe}^{2+}$ and subsequent oxidation (sample VI): (b) untreated holoferritin (sample I). Magnetic hyperfine field distributions are shown to the right of each spectrum.

quadrupole doublet and magnetic hyperfine sextet, there was an additional doublet with isomer shift and quadrupole splitting characteristic of high-spin $\mathrm{Fe}^{2+}$ in the core. At $80 \mathrm{~K}$, the relative absorption intensities of the ferric and ferrous doublets were approximately $3: 2$, corresponding to $40 \%$ reduction of the ferric core particle. Below $60 \mathrm{~K}$ the ferric subspectrum underwent a superparamagnetic transition as in fully oxidized ferritin. The magnetic fraction of the ferric subspectrum of sample $\mathrm{V}$ is also plotted as a function of temperature in fig. 1. It can be seen that partial reduction of the core particles without removal of the $\mathrm{Fe}^{2+}$ fraction resulted in an increase in the average blocking temperature compared to $40 \%$ reduction of the core particle followed by removal of the resulting $\mathrm{Fe}^{2+}$ (sample III).

The $2 \mathrm{~K}$ spectrum of sample $\mathrm{VI}$ is shown together with that for holoferritin (sample I) in fig. 3. Computer analysis showed that the low temperature sextet spectral parameters of the two samples were very similar, including the magnitude and sign of the quadrupole shift. This was also true for the parameters of the 


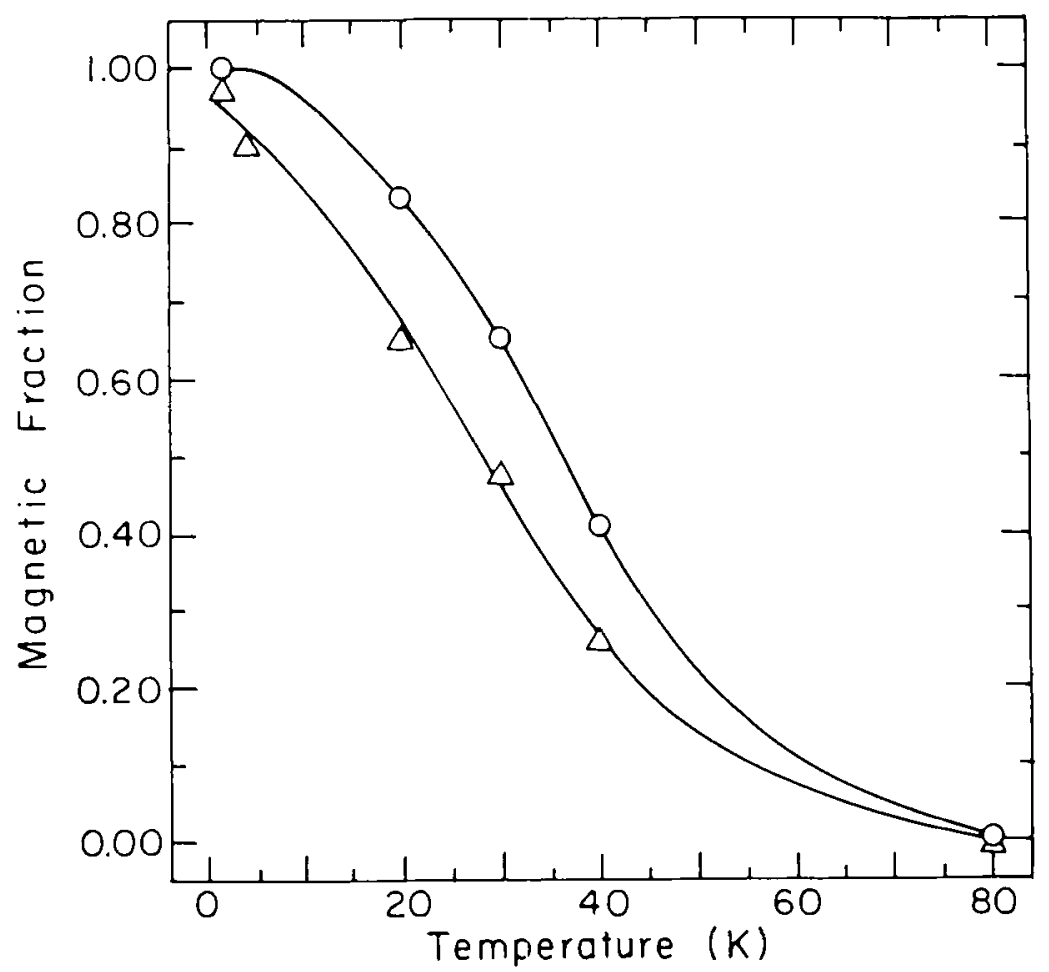

Fig. 4. Magnetic fractions plotted as a function of temperature for holoferritin (sample I) ( $)$ ) and holoferritin following incubation with ${ }^{57} \mathrm{Fe}^{2+}$ and subsequent oxidation (sample V1) $(\Delta)$.

high temperature quadrupole doublet. However, as can be seen in fig. 3, the lines in the sample VI spectrum were broader than the lines in the sample I spectrum, indicating a broader distribution of hyperfine fields in sample VI. Moreover, as shown in fig. 4, the average blocking temperature of sample VI was lower than that of sample I.

\section{Discussion}

According to the data in figs. 1 and 2 , the average blocking temperature decreases as iron is removed from the core. This result is consistent with measurements on ferritin samples fractionated by iron content [26]. It is also consistent with the theory of superparamagnetism applied to ferritin. According to eqs. (1) and (2), the blocking temperature should be linearly dependent on $n$, the number of iron atoms in the core, assuming the volume increases linearly with $n$, and the anisotropy energy per unit volume is a constant. However, the data in fig. 2 show that $\left\langle T_{\mathrm{B}}\right\rangle$ decreased much more slowly than linearly with decreasing n. This experimental result requires that the average anisotropy energy per iron 
atom. $e_{\mathrm{A}}=E_{\mathrm{A}} / n$, increase as the number of iron atoms decrease. This can be accounted for qualitatively by assuming that the anisotropy energy contributed by each iron atom depends on whether it is a bulk iron atom. in the interior of the core particle, or a surface iron atom, on the surface of the core particle. The distinction is reasonable considering that surface iron atoms are structurally more anisotropic than bulk iron atoms. For a Euclidean solid, the number of surface iron atoms, $n_{\mathrm{s}}$, is given by

$n_{\mathrm{s}}=a n^{2 / 3}$.

where $a$ is a constant that depends on the shape of the core solid. For a cube and a sphere, $a$ is 6 and 4 , respectively. Since the core particle is probably an irregular solid we assume $a=5$. Then the average anisotropy energy per iron atom $e_{\mathrm{A}}$ is given by

$e_{\mathrm{A}}=\frac{n_{\mathrm{s}} K_{\mathrm{s}}+n_{\mathrm{B}} K_{\mathrm{B}}}{n}$.

where $n_{\mathrm{B}}$ is the number of bulk iron atoms, $K_{\mathrm{s}}$ and $K_{\mathrm{B}}$ are the respective anistropy contributions and $n=n_{\mathrm{s}}+n_{\mathrm{B}}$. The blocking temperature is defined by eq. (1) with $\nu=\nu_{\mathrm{L}}$, the Larmor precession frequency $\left(10^{8} \mathrm{~s}^{-1}\right)$ :

$T_{\mathrm{B}}=e_{\mathrm{A}} n / 2.3 k(K)$.

Using the experimental blocking temperatures (table 1 ), $K_{\mathrm{B}} / k=-0.0493(\mathrm{~K})$ and $K / k=0.183(\mathrm{~K})$. The solid curve in fig. 2 was drawn using these values. For $n \leqslant 125$, all the iron atoms are on the surface of the core particle and $\left\langle T_{\mathrm{B}}\right\rangle$ is given by

$\left\langle T_{\mathrm{B}}\right\rangle=0.080 n(K)(n \leqslant 125)$.

Table 1

Measured and calculated blocking temperatures

\begin{tabular}{|c|c|c|c|c|c|c|}
\hline Simple & $n^{i}$ & $\left\langle T_{\mathrm{B}}\right\rangle(\mathrm{K})^{\mathrm{h}}$ & $n_{\mathrm{S}}{ }^{\mathrm{s}}$ & $n_{\mathrm{B}}{ }^{\circ}$ & $e_{\mathrm{A}} / k(\mathrm{~K})^{\mathrm{d}}$ & $T_{\mathrm{B}}(\mathrm{K}){ }^{\circ}$ \\
\hline I & 2100 & 38 & 822 & 1278 & 0.0416 & 38.0 \\
\hline II & 1680 & 36 & 708 & 972 & 0.0486 & 35.5 \\
\hline III & 1260 & 34 & 585 & 675 & 0.0586 & 32.1 \\
\hline IV & 840 & 27 & 446 & 394 & 0.0740 & 27.0 \\
\hline \multirow[t]{3}{*}{ V } & $2100^{r}$ & 51 & & & & \\
\hline & $4500^{\circ}$ & & 1367 & 3133 & 0.0213 & 41.6 \\
\hline & $125^{\circ}$ & & 125 & 0 & 0.183 & 9.9 \\
\hline
\end{tabular}

Total iron atoms per ferritin molecule.

${ }^{b}$ Experimental average blocking temperature.

" $n_{\mathrm{s}}=$ Surface iron atoms; $n_{\mathrm{B}}=$ Bulk iron atoms.

"Average anisotropy energy per iron atom (eq. (4)) with $K_{\checkmark} / k=0.183 \mathrm{~K}, K_{\mathrm{B}} / k=-0.0493 \mathrm{~K}$.

"Calculated blocking temperature (eq. (5)).

$40 \% \mathrm{Fe}^{2+} \cdot 60 \% \mathrm{Fe}^{3+}$.

'Calculated only. 
The sign difference for the bulk and surface contributions might indicate that the respective spin orientations are mutually perpendicular. Non-colinear spin arrangements in small ferrimagnetic particles, due to canting of the surface spins, have been reported [32].

Because the growth of the core particle might be limited by diffusion of ions into the ferritin core, the core particle might be a non-Euclidean solid. Diffusionlimited aggregation (DLA) processes result in fractal solids with surface dimension $d>2$ [33]. Computer models of DLA processes give $d=2.5$ [34]. If the core particles are indeed DLA structures, the number of iron atoms on the surface will be a higher power of the total number of iron atoms than given by eq. (3). For $d=2.5$,

$n_{\mathrm{S}}=b n^{0.833}$,

where $b$ is a constant. For a given value of $b$, values can be obtained for $K_{\mathrm{B}} / k$ and $K_{\mathrm{S}} / k$. Thus the dependence of the blocking temperature on iron loading alone is not sufficient to discriminate between Euclidean and fractal core particles.

The increase in $\left\langle T_{\mathrm{B}}\right\rangle$ for sample $\mathrm{V}$, with 1260 ferric ions and 840 ferrous ions per core particle, compared to sample IV, with 1260 ferric ions and no ferrous ions per core particle, can be understood in terms of a model in which the ferric ions in sample $\mathrm{V}$ form a contiguous particle similar to that in sample IV, but with the ferrous ions on or near the surface [35]. Because of unquenched orbital angular momentum they could affect the magnetic properties of the core ferric ions. Thus we could assume that the anisotropy contribution of the bulk ferric ions in sample $\mathrm{V}$ is the same as in sample IV, but the anisotropy contribution of ferric ions exchange coupled to ferrous ions in sample $\mathrm{V}$ is increased over that of the ferric ions in sample IV.

The calculated magnetic anisotropy energy for a totally filled ferritin core (4.500 iron atoms, $70 \AA$ diameter, table 1) corresponds to an anisotropy energy per unit volume of $5.6 \times 10^{4} \mathrm{erg} / \mathrm{cm}^{3}$. This value is consistent with those previously reported for ferritin. For example, combined Mössbauer and electron microscope studies on ferritin [21] yield anisotropy constants of the order of $1 \times 10^{5} \mathrm{erg} / \mathrm{cm}^{3}$, while a value of $6.7 \times 10^{4} \mathrm{erg} / \mathrm{cm}^{3}$ has been deduced from an analysis of the low temperature Mössbauer line shapes based on a modification of the superparamagnetic theory to include collective excitations [20].

An increase of up to two orders of magnitude in the anistropy energies of small particles of $\alpha-\mathrm{Fe}_{2} \mathrm{O}_{3}$ and $\mathrm{Fe}_{3} \mathrm{O}_{4}$ compared to the bulk magnetocrystalline anisotropies of these materials has been reported [36-39]. Furthermore, the anisotropy energy increases as the particle size decreases [40,41]. The increase in anisotropy has been attributed to the contribution of shape, surface and stress anisotropies present in small particles in addition to the crystalline anisotropy of the material. Shape anisotropy contributions are expected in the case of ferromagnetic or ferrimagnetic small particles [39] due to different demagnetizing 
factors along different directions of a spheroid particle [42]. Due to the low inherent magnetization of ferritin [14-16], the shape contribution to the anisotropy is expected to be small, and since any stress exerted by the surrounding protein shell on the iron core particle is probably isotropic due to the spherical shape of the protein shell, we have primarily considered surface effects. It was first pointed out by Néel [43] and later verified experimentally [44], that surface effects can contribute to the anisotropy. In addition, different values of the anisotropy energy constant in unsupported and supported $\alpha-\mathrm{Fe}_{2} \mathrm{O}_{3}$ microcrystals have been found [45.46]. and the adsorption of different molecules on $6 \mathrm{~nm} \mathrm{Fe} \mathrm{O}_{4}$ particles has been observed to result in different superparamagnetic relaxation times [37]. The latter observation is consistent with the increase of the anisotropy energy when ferrous ions were situated on or near the surface of the ferritin core particles.

Williams et al. [20] compared the magnetic hyperfine field distribution in holoferritin with that in holoferritin which had been incubated with ferric ions enriched in ${ }^{57} \mathrm{Fe}$. They found that the mode of the distribution was $495 \mathrm{kOe}$ in both cases, but that the distribution was broader in holoferritin, corresponding to a larger contribution from smaller particles in holoferritin. In the present experiments, the mode of the magnetic hyperfine field distribution was the same for samples VI and holoferritin (sample I), but sample VI had a broader distribution than holoferritin (fig. 3). This could be due to different methods of addition of ferric ions to the core, i.e., incubation with ferric ions [20], or, anaerobic incubation with ferrous ions followed by air oxidation (present experiments). These conditions, or incubation with ferrous iron in the presence of air [23.47]. could affect the distribution of ferric ions in the ferritin cores. Other factors affecting distribution might include $\mathrm{pH}$, phosphate content. and ageing (i.e., loss of water and rearrangement of ions) of the core particles.

In addition to a broader hyperfine field distribution, sample VI had lower average blocking temperature than holoferritin, although sample VI had a larger ferric iron content than holoferritin. There are at least three ways to interpret this result: 1) the ${ }^{57} \mathrm{Fe}^{2+}$ was predominantly taken up by molecules with less iron than average when ferrous ions were incubated with holoferritin, so that after air oxidation the "last-in" ferric ions were situated primarily in the less loaded ferritin molecules; 2) the "last-in" ferric ions were distributed somewhat uniformly among the ferritin molecules, and were situated on the surfaces of the holoferritin core particles, but relaxed at a different rate than the bulk ferric ions: 3) the "last-in" ferric ions were not uniformly situated on the surfaces of the holoferritin core particles, but tended to form. or form on, smaller clusters or domains within the core. as expected for a DLA process.

While the data presented here do not unequivocally support any one of the three interpretations advanced above, they best match interpretation 3. Interpretation 1 is not valid because we have found (Watt et al., unpublished) that ferritin molecules with more ferric ions in the core take up more ferrous ions under 
anaerobic conditions than less filled molecules. Interpretation 2 requires a radical reformulation of superparamagnetic relaxation theory and seems unlikely. In contrast, the lower blocking temperature, the broader magnetic hyperfine field distribution, and the remaining quadrupole doublet in the $2 \mathrm{~K}$ spectrum of sample VI (about 3\% of the spectral area) are all consistent with interpretation 3, with the "last-in" ferric ions forming. or forming on. smaller clusters or domains within the ferritin core.

In conclusion, the experiments presented here show that the magnetic anisotropy of ferritin core particles is a function of particle volume, and we have suggested a simple model based on surface and bulk contributions to the magnetic anisotropy per ferric ion in the particle. They further suggest that the "last-in" ferric ions form, or form on. smaller clusters or domains within the ferritin core.

\section{Acknowledgements}

RBF and GDW were supported by the National Institutes of Health. GCP was supported by the Office of Naval Research.

\section{References}

[1] G.C. Ford, P.M. Harrison. D.W. Rice, J.M.A. Smith. A. Treffrey, J.L. White and J. Yariv. Phil. Trans. R. Soc. Lond. B 304 (1984) 551.

[2] F.C. Theil. Ann. Rev. Biochem. 56 (1987) 289.

[3] E.I. Steifel and G.D. Watt. Nature (Lond.) 279 (1979) 81.

[4] E.R. Bauminger, S.G. Cohen, S. Ofer and E.A. Rachmilewitz. Proc. Natl. Acad. Sci. (USA) 76 (1979) 939.

[5] P.J. Artymiuk. E.R. Bauminger. P.M. Harrison. D.M. Lawson. I. Nowik, A.Treffrey and S.J. Yewdall, in: Iron Biominerals, eds. R.B. Frankel and R.P. Blakemore (Plenum Press, New York, 1990) 269.

[6] P.M. Harrison. F.A. Fischback. T.G. Hoy and G.H. Haggis, Nature (Lond.) 216 (1967) 1188.

[7] K.M. Towe, J. Biol. Chem. 256 (1981) 9377.

[8] W.H. Massover and J.M. Cowley. Proc. Natl. Acad. Sci. (USA) 70 (1973) 3847.

[9] S. Mann, J.V. Bannister and R.J.P. Williams. J. Mol. Biol. 188 (1986) 225.

[10] C. Yang. A. Meagher, B.H. Huynh, D.E. Sayers and E.C. Theil, Biochemistry 26 (1987) 497.

[11] M.P. Weir, T.J. Peters and J.F. Gibson. Biochim. Biophys. Actu 828 (1985) 298.

[12] S.H. Koenig. C.M. Doglin and R.D. Brown. Magn. Reson. Med. 2 (1985) 283.

[1.3] S. H. Koenig. R.D. Brown. J.F. Gibson. R.J. Ward and T.J. Peters. Magn. Reson. Med. 3 (1986) 755 .

[14] A. Blaise. J. Chappert and J.L. Girardet. C.R. Acad. Sci. (Paris) 261 (1965) 2310.

[15] A. Blaise, J. Feron. J.L. Giradet and J.J. Lawrence, C.R. Acad. Sci. (Paris) 265 (1967) 1077.

[16] J.L. Girardet, A. Blaise, J. Chappert, J.J. Lawrence. J. Feron and J.C. Picoche, J. Appl. Phys. 41 (1970) 1002 .

[17] A. Blaise and J.L Girardet. Proc. Int. Conf. on Magnetism. Moscow, 1973.

[18] J.F. Boas and B. Window. Aust. J. Phys. 19 (1966) 573. 
[19] W.T. Oosterhuis and K. Spartalian, in: Applications of Mössbauer Spectroscopy, ed. R.L. Cohen. Vol. 1 (Academic Press, NY, 1976) 142.

[20] J.M. Williams, D.P. Danson and Chr. Janot, Phys. Med. Biol. 23 (1978) 835.

[21] S.H. Bell, M.P. Weir. D.P.E. Dickson, J.F. Gibson, G.A. Sharp and T.J. Peters, Biochem. Biophys. Acta 787 (1984) 227.

[22] G.D. Watt. R.B. Frankel and G.C. Papaefthymiou. Proc. Natl. Acad. Sci. (USA) 82 (1985) 3640 .

[23] E.R.Bauminger. P.M. Harrison. I. Nowik and A. Treffry. Biochemistry 28 (1989) 5486.

[24] T.G. St. Pierre, S.H. Bell, D.P.E. Dickson, S. Mann, J. Webb, G.R. Moore and R.J.P. Williams, Biochim. Biophys. Acta 870 (1986) 127.

[25] T.G. St. Pierre. D.H. Jones and D.P.E. Dickson. J. Magn. Magn. Mater. 69 (1987) 276.

[26] T.G. St. Pierre, R.K. Pollard, D.P.E. Dickson. R.J. Ward and T.J. Peters, Biochim. Biophys. Acta 952 (1988) 158.

[27] L. Néel. J. Phys. Soc. Japan. Suppl. B-1 17 (1962) 676.

[28] B. Rodmacq. J. Phys. Chem. Solids 45 (1984) 1119.

[29] D.G. Rancourt and J.M. Daniels, Phys. Rev. B. 29 (1984) 2410.

[30] D.L. Jacobs, G.D. Watt. R.B. Frankel and G.C. Papaefthymiou. Biochemistry 28 (1989) 9216.

[31] D.L. Jacohs, G.D. Watt. R.B. Frankel and G.C. Papaefthymiou, Biochemistry 28 (1989) 1650.

[32] J.M.D. Coey, Phys. Rev. Lett. 27 (1971) 1140.

[33] R.M. Bradley and R.C. Ball, Nature 309 (1984) 225.

[34] P. Meakin, Phys. Rev. A227 (1985) 604.

[35] R.B. Frankel, G.C. Papaefthymiou and G.D. Watt, Hyp. Int. 33 (1987) 233.

[36] L.R. Bickford, J.M. Brownlow and R.F. Penoyer, Proc. Inst. Elec. Eng. B104 (1957) 238.

[37] S. Morup, H. Topsøe and J. Lipka. J. Physique. 37 (1976) C6-287.

[38] C.J.W. Koch. M.B. Madsen. S. Morup. L. Christiansen, L. Gerward and J. Willadsen. Clays and Clay Minerals 34 (1986) 17.

[39] T.K. McNab, R.A. Fox and A.J.F. Boyle, J. Appl. Phys. 39 (1968) 5703.

[40] S. Mørup and H. Topsøe. Appl. Phys. 11 (1976) 63.

[41] S. Morup. J.A. Dumesic and H. Topsøe, in: Applications of Mösshauer Spectroscopy. Vol. II. ed. R.L. Cohen (1980) 1.

[42] J.A. Osborn. Phys. Rev. 67 (1945) 67.

[43] L. Néel, J. Physique Radium 15 (1954) 225.

[44] A.E. Berkowitz. J.A. Lahut, I.S. Jacobs, L.M. Levinson and D.W. Forester, Phys. Rev. Lett. 34 (1975) 594.

[45] J.M.D. Coey and D. Khalafalla. Phys. Status Solidi 11 (1972) 229.

[46] M. Boudart, J.A. Dumesic and H. Topsae, Proc. Nat. Acad. Sci. (USA) 74 (1977) 806.

[47] J.S. Rohrer. R.B. Frankel. G.C. Papaefthymiou and E.C. Theil, Inorganic Chem. 28 (1989) 3393. 